\title{
An explicit expression for finite-size corrections to the chemical potential
}

\author{
B Smit $\dagger$ and D Frenkel $\ddagger$ \\ $\dagger$ Koninklijke/Shell-Laboratorium, Amsterdam (Shell Research BV), Badhuisweg 3, 1031 \\ CM Amsterdam, The Netherlands \\ $\doteqdot$ FOM Institute for Atomic and Molecular Physics, Kruislaan 407, 1098 SJ Amsterdam, \\ The Netherlands
}

Received 10 July 1989

\begin{abstract}
In this article an expression is derived for the finite-size corrections to the excess chemical potential in an $N$-particle system with periodic boundary conditions. The leading $N$-dependence of the chemical potential is predicted to be proportional to $1 / N$. We derive a simple expression relating the coefficient of this $1 / N$-term to the compressibility of the system.

In order to test the present predictions, Monte Carlo simulations were performed in which the chemical potential of the hard-sphere fluid and of the Lennard-Jones fluid were calculated using the Widom particle insertion method. The results of these simulations are in excellent agreement with the predicted $N$-dependence.
\end{abstract}

\section{Introduction}

The chemical potential of a (one-component) system is equal to the change of the free energy caused by the addition of a particle to the system. An explicit expression for the excess chemical potential $\mu^{\text {ex }}$ of a system at constant $N, V, T$ was derived by Widom [1] in 1963. This expression relates $\mu^{\text {ex }}$ to the average value of the Boltzmann factor $\exp \left(-\beta \Delta U^{+}\right)$associated with the random insertion of a test particle into the $N, V, T$ system:

$$
\mu^{\mathrm{ex}}=-(1 / \beta) \log \left(\exp \left(-\beta \Delta U^{+}\right)\right\rangle_{N, V, T}
$$

where $\Delta U^{+}$is the interaction energy of the test particle with the remaining $N$ particles.

Equation (1) can be used to compute the excess chemical potential in a $N, V, T$ Monte Carlo simulation. This was first done by Adams [2] who applied (1) to a fluid of hard spheres. Another atomic system that has been studied extensively using the Widom method is the Lennard-Jones fluid [3-5]. Widom's original expression (equation (1)) was derived for the $N, V, T$ ensemble. The corresponding expression for the excess chemical potential in the $N, V, E$ ensemble is derived in [6], for the $N, P, T$ ensemble in $[7,8]$, and for the Gibbs ensemble in [9].

The excess chemical potential measured by the Widom particle insertion method exhibits a strong $N$-dependence. This fact has been pointed out by many authors, starting with Adams [2], and more recently by Heinbuch and Fischer [4] for the Lennard-Jones fluid. However, to our knowledge, a quantitative estimate of this $N$-dependence is lacking. One reason is that there are in fact several mechanisms that contribute to the system 
size dependence of the chemical potential. In this article we derive an expression that predicts a $1 / N$-dependence for what we believe to be the most important corrections to the excess chemical potential in finite systems. The derivation of this expression is given in the next section. In $\S 3$ this expression is compared with Monte Carlo results for a hard-sphere fluid and Lennard-Jones fluid.

\section{Particle insertion in an infinite periodic system}

Our derivation of the finite-size corrections to the excess chemical potential $\left(\mu^{\mathrm{ex}}\right)$ is based on an elegant derivation of the Ornstein-Zernike compressibility relation that was recently given by Vrij [10]. The basic idea behind Vrij's derivation of the OrnsteinZernike equation [11] is the following. Consider a fluid in a large but finite volume $V$ separated by a semi-permeable membrane from a large reservoir. The fluid particles can cross the membrane. Now we add to the volume $V$ a test particle, identical to the other fluid molecules, but for the fact that it cannot cross the membrane. This test particle will displace a certain number of fluid molecules. The total change in the number of fluid molecules in volume $V$ is given by $\nu$ :

$$
\nu=4 \pi \rho \int_{0}^{\infty} \mathrm{d} r r^{2} h(r)
$$

where $h(r)=g(r)-1$ and $\rho$ is the fluid density. Vrij then proceeds to derive the Ornstein-Zernike compressibility relation:

$$
1+\nu=k T(\partial \rho / \partial P)_{T} .
$$

Now consider the same insertion of a test particle in the context of the chemical potential. Again, we start from the usual definitions of the chemical potential $\mu=$ $(\partial F / \partial N)_{V, T}$. However, instead of relating $\mu$ to the average value of the Boltzmann factor of a test particle inserted at a random position in the system, it is now more convenient to interpret $\mu$ as the reversible work needed to add an additional particle to the system. In this picture, $\mu^{\mathrm{ex}}$ is equal to the reversible work that we must perform if we slowly switch on the interactions between this particle and the $N$ particles already present in the system. What is the difference if we insert the particle in a finite volume $V$ that is part of an infinite periodic system? The main difference with the infinite-system case is that no particles can flow out of volume $V$. This constraint increases the reversible work needed to insert a particle. The easiest way to estimate this excess reversible work needed to insert a particle is to consider a process where we first add a test particle to a volume $V$ in open contact with a reservoir. Next, we compute the reversible work $\Delta \mu \mu_{N}^{\text {ex }}$ needed to recompress the particles that were pushed out of the volume $V$ back into it. This additional reversible work is our estimate for the $N$-dependent correction to $\mu^{\mathrm{ex}}$.

Let us denote the number of particles that must be recompressed into the volume $V$ by $\Delta N$; then:

$$
\begin{gathered}
\Delta \mu_{N}^{\mathrm{ex}}=-\mu_{\text {bath }} \Delta N+(\partial F(N) / \partial N)_{V, T} \Delta N+\frac{1}{2}\left(\partial^{2} F(N) / \partial N^{2}\right)_{V, T} \Delta N^{2}+\ldots \\
=\frac{1}{2}\left(\partial^{2} F(N) / \partial N^{2}\right)_{V, T} \Delta N^{2}+\mathrm{O}\left(\Delta N^{3}\right)
\end{gathered}
$$

where $F(N)$ is the Helmholtz free energy of a system of $N$ particles in volume $V$ at temperature $T$, and where we have used the fact that in equilibrium the first and second terms on the right-hand side of the top line of (4) cancel. To lowest order in $\Delta N$ we find therefore that $\Delta \mu \mu_{N}^{\mathrm{ex}}$ is given by

$\Delta \mu_{N}^{\mathrm{ex}}=\frac{1}{2}\left(\partial^{2} F(N) / \partial N^{2}\right)_{V, T} \Delta N^{2}=\frac{1}{2}(\partial \mu / \partial N)_{V, T} \Delta N^{2}=(1 / 2 N)(\partial P / \partial \rho)_{V, T} \Delta N^{2}$.

In the last equality in (5) we have used the Gibbs-Duhem relation. Finally we use the 
fact that $\Delta N=\nu$ (see equation (2)) and we use the Ornstein-Zernike relation (equation (3)). We then find that to leading order in $1 / N$, the finite-size correction to the excess chemical potential is given by

$$
\Delta \mu_{N}^{\mathrm{ex}}=(1 / 2 N)(\partial P / \partial \rho)_{V, T}\left[1-k T(\partial \rho / \partial P)_{T}\right]^{2} .
$$

Note first of all that equation (6) does indeed predict a $1 / N$-dependence of the finitesize correction to the chemical potential and, more importantly, that this correction can be evaluated explicitly from knowledge of the compressibility of the system. Equation (6) is the central result of this paper. From this equation, the leading $N$-dependence in other thermodynamic quantities, such as $F^{\mathrm{ex}}$ and $P^{\mathrm{ex}}$ is easily derived. In particular, to derive the corresponding finite-size corrections to the excess Helmholtz free energy $F^{\mathrm{ex}}$, we use the thermodynamic relation

$$
\left[\left(\partial F^{\mathrm{ex}} / V\right) /(\partial 1 / V)\right]_{N, T}=\left(\partial \rho F^{\mathrm{ex}} / \partial \rho\right)_{N, T}=G^{\mathrm{ex}}
$$

for a one-component system where $G^{\mathrm{ex}}=\mu^{\mathrm{ex}}$, the finite-size correction to $F^{\mathrm{ex}}, \Delta F_{N}^{\mathrm{ex}}$ is related to $\Delta \mu_{N}^{\text {ex }}$ in (6) by

$$
\left(\partial \rho \Delta F_{N}^{\mathrm{ex}} / \partial \rho\right)_{N, T}=(1 / 2 N)(\partial P / \partial \rho)_{V, T}\left[1-k T(\partial \rho / \partial P)_{T}\right]^{2} .
$$

At $\rho=0, \Delta F_{N}^{\mathrm{ex}}$ vanishes. Hence we can compute $\Delta F_{N}^{\mathrm{ex}}$ at finite $\rho$ simply by integrating from $\rho=0$ :

$$
\begin{gathered}
\Delta F_{N}^{\mathrm{ex}}(\rho)=\frac{1}{2 N \rho} \int_{0}^{\rho} \mathrm{d} \rho\left(\frac{\partial P}{\partial \rho}\right)_{V, T}\left[1-k T\left(\frac{\partial \rho}{\partial P}\right)_{T}\right]^{2} \\
=\frac{1}{2 N \rho} \int_{0}^{P} \mathrm{~d} P\left[1-k T\left(\frac{\partial \rho}{\partial P}\right)_{T}\right]^{2}
\end{gathered}
$$

Given the equation of state, equation (9) yields an explicit expression for $\Delta F_{N}^{\mathrm{ex}}$. This expression in turn can be used to compute the finite-size corrections to $P^{\mathrm{ex}}=P-P^{\text {id }}$.

\section{Comparison with computer simulations}

In order to test the expression for the $N$-dependence of the excess chemical potential for finite periodic systems, as derived in the previous section, we performed Monte Carlo simulations in the $N, V, T$ ensemble on a hard-sphere fluid and a Lennard-Jones fluid.

\subsection{Hard-sphere fluid}

In the case of a hard-sphere fluid the pair potential is given by

$$
\Phi(r)= \begin{cases}\infty & r<\sigma \\ 0 & r \geqslant \sigma .\end{cases}
$$

Therefore the energy of a test particle is either $\infty$ or 0 and $\left\langle\exp -\left(-\beta \Delta U^{+}\right)\right\rangle$is equal to the probability $P$ of placing a sphere into the system without overlapping with one of the spheres already present in the system. The excess chemical potential is then simply given by

$$
\beta \mu_{\text {excess }}=-\log (P) \text {. }
$$

The Monte Carlo simulations were started from an FCC lattice $(N=32$ and $N=108)$ 
Table 1. Summary of the Monte Carlo results for the excess chemical potential of the hardsphere fluid. $\rho^{*}$ is the reduced density, $N$ is the number of particles, $N_{\mathrm{MC}}$ is the number of Monte Carlo steps, and $N_{\text {try }}$ is the number of attempts to insert a test particle. $\mu^{\text {ex }}$ is the excess chemical potential. The figure in parentheses is the accuracy of the last digit(s), so 3.894(11) means $3.894 \pm 0.11$.

\begin{tabular}{lrrrll}
\hline$\rho^{*}$ & \multicolumn{1}{l}{$N$} & $10^{-6} N_{\text {MC }}$ & $N_{\text {try }}$ & $\beta \mu^{\text {ex }}$ & $\beta \mu^{\text {ex }}[2]$ \\
\hline 0.5 & 32 & 6.4 & 20 & $3.894(11)$ & 3.885 \\
& 64 & 13.4 & 80 & $3.856(09)$ & \\
& 108 & 5.4 & 120 & $3.841(10)$ & \\
& 256 & & & & 3.832 \\
0.6 & 32 & 16.0 & 30 & $5.490(11)$ & 5.457 \\
& 64 & 13.4 & 50 & $5.414(25)$ & \\
& 108 & 5.4 & 80 & $5.387(18)$ & \\
& 256 & & & & 5.359 \\
0.7 & 32 & 12.0 & 60 & $7.645(20)$ & 7.669 \\
& 64 & 16.0 & 100 & $7.515(40)$ & \\
& 108 & 32.0 & 180 & $7.434(19)$ & \\
& 256 & & & & 7.391 \\
& 864 & & & & 7.371 \\
\hline
\end{tabular}

or from a simple cubic lattice $(N=64)$. Cubic periodic boundary conditions were used. One Monte Carlo step is defined as one attempt to move a particle. The maximum displacement for one Monte Carlo step is chosen in such a way that approximately $30 \%$ of the Monte Carlo steps were accepted. After equilibration the Monte Carlo process consists of the following steps: first one attempt to move each particle successively followed by $N_{\text {try }}$ attempts to insert a test particle at a random position in the simulation volume. The accuracy of the simulations was estimated by dividing the total simulation into sub-runs. The standard deviations obtained from these block averages were used to estimate the standard deviations in the chemical potentials.

In table 1 the results of the Monte Carlo simulations for $\rho^{*}=0.5,0.6$ and 0.7 are summarised. Comparison with the results of Adams [2] shows reasonable agreement. In figure 1 the $N$-dependence of the chemical potential is shown. These results indicate a $1 /$ $N$-dependence of the finite-size corrections to the chemical potential, as expected. As can be seen from figure 1 , the magnitude of the finite-size correction increases with density. Note also that these finite-size effects are by no means small for systems with $N=\mathrm{O}\left(10^{2}\right)$.

In order to make a quantitative comparison with (6) we have used the CarnahanStarling [11] equation to calculate the compressibility of the hard-sphere fluid. In table 2 and in figure 1 the results of these calculations are compared with the Monte Carlo simulations. The agreement of the Monte Carlo data with the predictions of equation (6) is excellent.

\subsection{Lennard-Jones fluid}

The Monte Carlo simulations for the Lennard-Jones fluid were started from a FCc lattice. The maximum displacement of one Monte Carlo step was chosen in such a way that approximately $40 \%$ of the attempts were accepted. Cubic periodic boundary conditions were used. After equilibration the Monte Carlo simulations were performed in two steps: first one attempt to move each particle successively followed by $N_{\text {try }}$ attempts to 


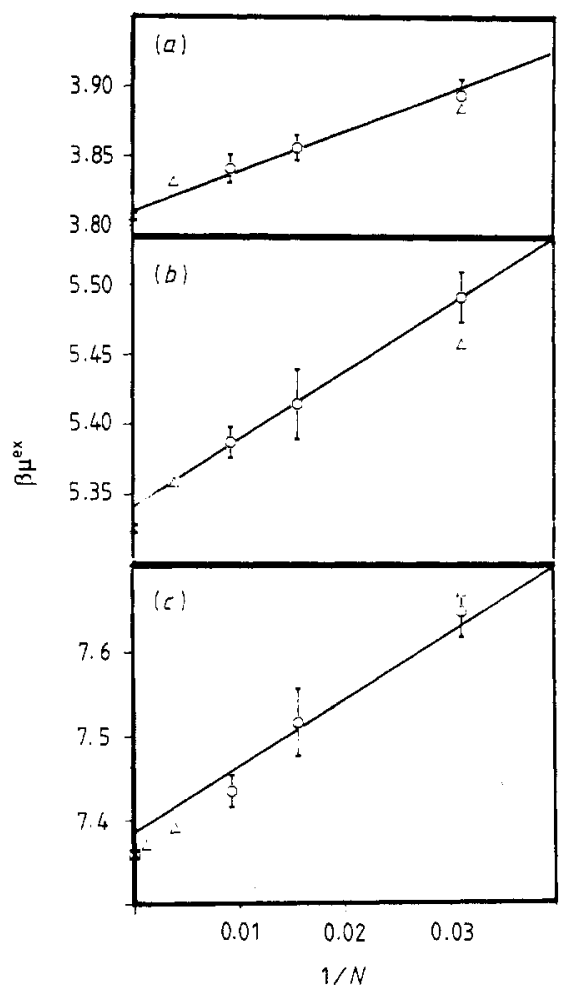

Figure 1. The excess chemical potential $\left(\beta \mu^{e x}\right)$ of a hard-sphere fluid as a function of the reciprocal number of particles $(1 / N)$, for $\rho^{*}=(a) 0.5,(b)$ 0.6 , and $(c) 0.7$. The lines are given by $\beta \mu^{\mathrm{ex}}(N)=$ $A / N+B$, where $A$ is obtained from (6) and $B$ is obtained from fitting to the Monte Carlo results. Key: $\bigcirc$, this work; $\triangle$, Adams; $\otimes$, CarnahanStarling.

Table 2. Comparison of the predicted $1 / N$-dependence of the excess chemical potential for a hard-sphere fluid with the Monte Carlo results for various densities. $\rho^{*}\left(=\rho \sigma^{3}\right)$ denotes the reduced density, $\Delta \mu_{N}^{\operatorname{exCS}}$ (equation (6)) was calculated using the Carnahan-Starling equation of state, and $\Delta \mu_{N}^{\text {exMC }}$ was obtained from a linear fit to the Monte Carlo data given in table 1.

\begin{tabular}{lll}
\hline$\rho^{*}$ & $N \Delta \mu_{N}^{\mathrm{exCS}}$ & $N \Delta \mu_{N}^{\mathrm{exMC}}$ \\
\hline 0.5 & 2.9 & $2.3 \pm 0.6$ \\
0.6 & 4.8 & $4.7 \pm 0.9$ \\
0.7 & 7.8 & $9.6 \pm 1.6$ \\
\hline
\end{tabular}

insert a test particle at a random position. The standard deviations of the chemical potential were estimated by dividing the total run into sub-runs and calculating block averages.

Tail corrections were applied by assuming that $g(r)=1$ for distances larger than the cut-off radius $R^{c}$. We have used half the box size as the cut-off radius. The results of Heinbuch and Fischer [4] suggest that the value of the chemical potential depends strongly on the cut-off radius. Therefore, we have performed several simulations for $N=256$ and for $N=500$ using smaller cut-off radii $\left(0.5(108 / \rho)^{1 / 3}<R^{\mathrm{c}}<0.5(N / \rho)^{1 / 3}\right)$. Within the accuracy of the calculations we could not observe any significant influence of the cut-off radius on our results for the chemical potential.

In table 3 the results of our Monte Carlo simulations for $\rho^{*}=0.6,0.7$ and 0.8 are summarised. Our results are in very good agreement with the results of Deitrick and coworkers [5]. However, there is a systematic difference from the results of Heinbuch and Fischer [4], which appears to be approximately half the long-tail correction. When this 
Table 3. Summary of the Monte Carlo results for the excess chemical potential of the Lennard-Jones fluid at $T^{*}=1.2 . \rho^{*}$ is the reduced density, $N$ is the number of particles, $N_{\mathrm{MC}}$ is the number of Monte Carlo steps and $\mu^{\mathrm{ex}}$ is the excess chemical potential. The value of the excess chemical potential in curly brackets is the corrected value (see the text). The figure in parentheses is the accuracy of the last digit(s).

\begin{tabular}{|c|c|c|c|c|c|c|}
\hline$\rho^{*}$ & $N$ & $10^{-6} N_{\mathrm{MC}}$ & $N_{\text {try }}$ & $\beta \mu^{\mathrm{ex}}$ (this work) & $\beta \mu^{\mathrm{ex}}[4]$ & $\beta \mu^{e x}[5]$ \\
\hline 0.20 & $\begin{array}{r}32 \\
108 \\
500\end{array}$ & $\begin{array}{l}1.9 \\
8.6 \\
2.0\end{array}$ & $\begin{array}{r}50 \\
50 \\
1000\end{array}$ & $\begin{array}{l}-1.320(6) \\
-1.326(7) \\
-1.339(7)\end{array}$ & $-1.31\{-1.32\}$ & \\
\hline 0.45 & $\begin{array}{r}64 \\
108 \\
256\end{array}$ & $\begin{array}{l}1.9 \\
3.2 \\
2.3\end{array}$ & $\begin{array}{l}100 \\
125 \\
150\end{array}$ & $\begin{array}{l}-2.386(12) \\
-2.356(15) \\
-2.333(42)\end{array}$ & & \\
\hline 0.6 & $\begin{array}{l}108 \\
256 \\
500\end{array}$ & $\begin{array}{l}5.4 \\
2.6 \\
2.5\end{array}$ & $\begin{array}{l}100 \\
200 \\
300\end{array}$ & $\begin{array}{l}-2.455(26) \\
-2.431(40) \\
-2.428(40)\end{array}$ & & \\
\hline 0.7 & $\begin{array}{l}108 \\
200 \\
256 \\
500\end{array}$ & $\begin{array}{r}5.0 \\
\\
10.2 \\
5.0\end{array}$ & $\begin{array}{l}150 \\
200 \\
300\end{array}$ & $\begin{array}{l}-1.889(70) \\
-1.908(40) \\
-1.940(30)\end{array}$ & $\begin{array}{l}-1.68\{-1.84\} \\
-1.87\{-1.92\} \\
-1.86\{-1.89\}\end{array}$ & $\begin{array}{l}-1.884 \\
-1.902 \\
-1.90\end{array}$ \\
\hline 0.8 & $\begin{array}{l}108 \\
200 \\
256 \\
500\end{array}$ & $\begin{array}{r}10.8 \\
5.0 \\
2.0\end{array}$ & $\begin{array}{r}200 \\
\\
200 \\
1000\end{array}$ & $\begin{array}{l}-0.51(17) \\
-0.56(30) \\
-0.58(40)\end{array}$ & & $\begin{array}{l}-0.521 \\
-0.596 \\
-0.594\end{array}$ \\
\hline
\end{tabular}

Table 4. Comparison of the predicted $1 / N$-dependence of the excess chemical potential for a Lennard-Jones fluid with the Monte Carlo results for various densities. $\rho^{*}\left(=\rho \sigma^{3}\right)$ denotes

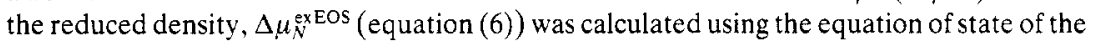
Lennard-Jones fluid given in [12], and $\Delta \mu_{N}^{\text {exMC }}$ is obtained from a linear fit to the Monte Carlo data given in table 3 .

\begin{tabular}{llc}
\hline$\rho^{*}$ & $N \Delta \mu_{N}^{\text {ex } \operatorname{EOS}}$ & $N \Delta \mu_{N}^{\text {exMC }}$ \\
\hline 0.20 & -5.1 & $0.5 \pm 0.3$ \\
0.45 & -2.5 & $-4.6 \pm 2.5$ \\
0.60 & 0.4 & $-3 \pm 4$ \\
0.70 & 2.6 & $7 \pm 6$ \\
0.80 & 6.6 & $9 \pm 7$ \\
\hline
\end{tabular}

correction is added to the data of [4] good agreement is obtained with the data of [5] and with our results. Furthermore, with this correction added the dependence on the cut-off radius noted in [4] disappears.

These results for the excess chemical potential show that compared with that of a hard-sphere fluid the $N$-dependence is much smaller. It is interesting to make a more quantitative comparison of these results with the predictions of equation (6). We have used the equation of state given by Nicolas and co-workers [12] to estimate the compressibility of the Lennard-Jones fluid. In table 4 the results of these calculations are compared with the Monte Carlo results. Because the $N$-dependence of the LennardJones fluid turned out to be much smaller it would require very extensive calculations to confirm the predictions of equation (6) with high accuracy. However, these data clearly demonstrate that (6) provides a good estimate for the finite-size corrections to the excess 
chemical potential for the Lennard-Jones fluid outside the two-phase region. Inside this region the present method is not supposed to work. For instance, equation (6) predicts that on the spinodal $((\partial P / \partial \rho)=0))$ the correction to $\Delta \mu^{\text {ex }}$ diverges. This is clearly an artefact of our implicit assumption that the dominant contribution to

$$
\int h(r) r^{2} \mathrm{~d} r
$$

comes from short-range correlations. This is clearly not correct at a spinodal. Nevertheless, it is amusing to note that in the region where $\partial P / \partial \rho<0$ in a finite system, we do indeed find evidence for a negative correction to $\mu^{\mathrm{ex}}$.

\section{Concluding remarks}

In this article we have derived an expression that predicts a $1 / N$-dependence of the finitesize corrections to the chemical potential of an infinite periodic system. Furthermore, comparison with results from Monte Carlo simulations shows that this expression correctly predicts the $N$-dependence of the excess chemical potential of the hard-sphere fluid and the Lennard-Jones fluid, as obtained by the Widom particle insertion method. Because this expression requires only knowledge of the compressibility of the system it can be used directly to correct the results of simulations on a small number of particles for finite-size effects. We believe that this result is of considerable practical importance for the location of phase coexistence lines by computer simulation. It should be stressed that, although the numerical test presented in this paper employed the Widom particle insertion method, equation (6) should apply to any measurement of the chemical potential in a periodic $N$-particle system.

\section{Acknowledgments}

The work of the FOM Institute is part of the research programme of FOM and is supported by the 'Nederlandse Organisatie voor Wetenschappelijk Onderzoek' (NWO). One of us (DF) would like to thank A Vrij for bringing [10] to his attention.

\section{References}

[1] Widom B 1963 J. Chem. Phys. 392802

[2] Adams D J 1974 Mol. Phys. 281252

[3] Shing K S and Gubbins K E 1982 Mol. Phys. 461109

Powles J G, Evans W A B and Quirke N 1982 Mol. Phys. 461347

Guilot B and Guissani Y 1985 Mol. Phys. 54455

[4] Heinbuch U and Fischer J 1987 Mol. Simul. 1109

[5] Deitrick G L, Scriven L E and Davis H T 1989 J. Chem. Phys. 902370

[6] Frenkel D 1985 Proc. 97th Int. 'Enrico Fermi' School of Physics (Varenna) 1985 ed. G Ciccotti and W G Hoover (Amsterdam: North-Holland) p 151

[7] Shing K S and Chung T S 1987 J. Phys. Chem. 911674

[8] Sindzingre P, Ciccotti G, Massobrio C and Frenkel D 1987 Chem. Phys. Lett. 13635

[9] Smit B and Frenkel D 1989 Mol. Phys. at press

[10] Vrij A 1985 Proc. Acad. Sci. Amsterdam B 88221

[11] Hansen J P and McDonald I R 1986 Theory of Simple Liquids 2nd edn (London: Academic)

[12] Nicolas J J, Gubbins K E, Streett W B and Tildesley D J 1979 Mol. Phys. 371429 